\title{
Quantifying test-retest reliability of repeated objective attentional measures in Lewy body dementia
}

\author{
Greg J. Elder ${ }^{1,2} \cdot$ Sean J. Colloby ${ }^{2} \cdot$ Michael J. Firbank $^{2} \cdot$ John-Paul Taylor $^{2}$
}

Received: 15 November 2021 / Revised: 12 January 2022 / Accepted: 18 January 2022 / Published online: 27 January 2022

(c) The Author(s) 2022

\begin{abstract}
Objective cognitive impairment is a feature of Lewy body dementia (LBD), and computerised attentional tasks are commonly used as outcome measures in interventional trials. However, the reliability of these measures, in the absence of interventions, are unknown. This study examined the reliability of these attentional measures at short-term and longer-term follow-up stages. LBD patients $(n=36)$ completed computerised attentional tasks [simple and choice reaction time, and digit vigilance (SRT, CRT, DV)] at short-term (Day 0-Day 5) and longer-term (4 and 12 weeks) follow-up. Intra-class correlations (ICCs) were calculated to assess test-retest reliability. At short-term, the reciprocal SRT, CRT and DV mean reaction time to correct answers, the reciprocal DV coefficient of variation, and reciprocal power of attention (PoA) all showed excellent levels of reliability (all ICCs $>0.90$ ). The reciprocal PoA showed the highest level of reliability (ICC $=0.978$ ). At longer-term followup, only the reciprocal PoA had excellent levels of reliability (ICC $=0.927)$. Reciprocal SRT, CRT and DV reaction time to correct answers, and the CRT coefficient of variation values, showed good levels of test-retest reliability (ICCs $\geq 0.85$ ). Contrary to expectations, most attentional measures demonstrated high levels of test-retest reliability at both short-term and longer-term follow-up time points. The reciprocal PoA composite measure demonstrated excellent levels of test-retest reliability, both in the short-term and long-term. This indicates that objective attentional tasks are suitable outcome measures in LBD studies and that the composite PoA measure may offer the highest levels of reliability.
\end{abstract}

Keywords Lewy body dementia $\cdot$ Attention $\cdot$ Reaction time $\cdot$ Trials $\cdot$ Outcome measures

\section{Background}

Lewy body dementia (LBD) is an umbrella term that refers to dementia with Lewy bodies (DLB) and Parkinson's disease dementia (PDD). DLB is a common cause of neurodegenerative dementia in older people and accounts for as much as $7.5 \%$ of diagnosed dementia cases in secondary care $[1,2]$. Dementia is frequently observed as a long-term outcome in Parkinson's disease (PD), since up to $80 \%$ of

Greg J. Elder and Sean J. Colloby are joint first authors.

Greg J. Elder

g.elder@northumbria.ac.uk

1 Northumbria Sleep Research, Department of Psychology, Faculty of Health and Life Sciences, Northumbria University, Newcastle upon Tyne NE1 8ST, UK

2 Translational and Clinical Research Institute, Newcastle University, Campus for Ageing and Vitality, Newcastle upon Tyne NE4 5PL, UK individuals with PD eventually develop dementia $[3,4]$. DLB and PDD share a common underlying alpha-synuclein neuropathology, and both dementias share the core diagnostic and clinical features of marked attentional and visuoperceptual deficits $[5,6]$.

Clinically, patients with LBD report impairments in subjective cognition. A common symptom is that of cognitive fluctuations, which refers to interruptions in awareness, reductions in alertness and transient episodes of confusion [7]. Objective impairments in cognition, measured using computerised reaction time tasks, are also apparent in LBD. Individuals with LBD display similar levels of objective attentional impairment, where this is more pronounced than is observed in Alzheimer's dementia (AD) [8, 9]. Compared to patients with $\mathrm{AD}$, those with $\mathrm{LBD}$ display slower reaction times (RTs) and higher levels of intra-individual variability [7, 10-12]. Attentional dysfunction can negatively affect quality of life in PDD [13] and may also contribute to other frequently-observed symptoms, including visual hallucinations [5, 14]. 
Objective measures of attention are frequently used as primary or secondary outcome measures in pharmacological and non-pharmacological LBD clinical trials [15-17]. This is also the case in ongoing, or planned, clinical trials, where objective attentional measures such as psychomotor speed, attention and memory, or composite objective measures (e.g. Continuity of Attention) are considered to be primary outcome measures (e.g. ClinicalTrials.gov; NCT03305809; NCT04739423). However, it is not currently known if measures of objective attention are stable when they are repeated at later time points, either in the short term, or over longer time spans, and it is important to understand this given the potential variance introduced by cognitive fluctuations. There is therefore a clear need to determine the stability of these outcome measures to validate their use and to aid the interpretation of these variables within the context of clinical trials $[2,18]$. This is particularly important when objective attentional or reaction time-based measures are assessed as primary outcomes.

The aim of the present study was to assess the test-retest reliability of objective attentional outcome measures in LBD between baseline and five consecutive days (shortterm follow-up), and between baseline and follow-up time points of 4 weeks and 12 weeks (longer-term follow-up). These time points are typical of those used in LBD clinical trials. The present study examined mean reaction time and the intra-individual variability in reaction times, as an objective marker of attentional fluctuations. This study also aimed to estimate the minimum number of trials needed to achieve acceptable levels of test-retest reliability, in order to potentially reduce task duration and intensity for future studies. Finally, this study also aimed to inform indicative sample sizes for LBD clinical trials where these measures are desired to be used as primary outcome measures.

It was hypothesised that individuals with LBD would demonstrate poor test-retest reliability in 1) overall reaction times; 2) intra-individual reaction time variability; 3 ) composite measures of focussed attention and central information processing speed. It was expected that this would be the case in the short-term and at longer-term follow-up time points.

\section{Methods}

\section{Participants}

Study participants were originally recruited to a randomised controlled clinical trial for an intervention to treat visual hallucinations (ISRCTN40214749) and therefore, all participants reported having visual hallucinations of a moderateto-severe nature prior to study entry. All data were obtained from study procedures conducted during the clinical trial.
Data were collected between November 2013 and December 2017. A total of 40 participants (DLB $n=26$, PDD $n=14$; $M_{\text {age }}=75.52$ years; $\mathrm{SD}_{\text {age }}=8.69$ years) were originally entered into the study and four participants dropped out of the study prior to the treatment week. Participants met diagnostic criteria for probable DLB or PDD [2, 5], as verified by two experienced clinicians.

Full inclusion and exclusion criteria, recruitment details and participant characteristics from this trial are reported in full elsewhere [19], but briefly, participants were recruited from clinical services in the North East of England and were $\geq 60$ years of age. Participants were enrolled in the study if they had no changes to relevant medication (e.g. anti-parkinsonian or psychotropic medication, or cholinesterase inhibitors) in the month preceding participation, had a Mini-Mental State Examination [MMSE; 20] score of $\geq 12$, and a sufficient level of English to allow for participation. Exclusion criteria included a history of excess alcohol intake, concurrent major psychiatric illness, and concurrent significant physical illness, co-morbidities or neurological disorders. All participants and their informants (participant carers/relatives) provided written informed consent and the study was approved by an NHS Research Ethics Committee (REC reference: 13/YH/0292).

\section{Control participant group}

Computerised task data were obtained from an additional group of 26 healthy control participants as a non-dementia comparison group ( 15 male, 11 female; $\mathrm{M}_{\text {age }}=74.38$ years; $\mathrm{SD}_{\mathrm{age}}=7.22$ years). These data were taken from two separate LBD observational studies, where participants were specifically recruited as comparator groups to dementia patients. All participants were recruited separately and provided written informed consent, including for the re-use of their anonymised data. Both studies were approved by an NHS Research Ethics Committee (REC references: 13/NE/0252; 13/NE/0359).

\section{Measures}

Part III of the Parkinson's Disease Rating Sale was used to assess motor function [21]. Global cognitive function was assessed using the MMSE [20] and Cambridge Cognitive Examination [CAMCOG; 22]. Memory and executive function domain subscores were also derived from the CAMCOG. Informants provided subjective ratings of the presence and severity of participant cognitive fluctuations using the One Day Fluctuation Scale (ODFAS) and Clinical Assessment of Fluctuation (CAF) scale [23], which assess cognitive fluctuations over the previous day and month respectively. Only the LBD patient group completed these measures. 


\section{Computerised attentional tasks}

Participants completed three attentional computerised tasks in the current study: simple reaction time (SRT), choice reaction time (CRT) and digit vigilance (DV) tasks, which have been previously shown to be differentially sensitive to LBD, relative to healthy control individuals and other dementias [24]. Tasks were programmed in MATLAB (Mathworks Inc) using the Cogent toolbox (www.vislab. ucl.ac.uk/cogent_2000.php). A laptop PC was used to present the tasks and participants responded using custom buttons held in the dominant hand (SRT, DV), or in both hands (CRT), depending on the task.

In the SRT task, a target (letter X) was displayed for a maximum of $3000 \mathrm{~ms}$ per trial, with a varying inter-stimulus interval, and participants were required to respond to the target as quickly as possible. In the CRT task, a target arrow which pointed left or right was displayed for a maximum of $3000 \mathrm{~ms}$ and participants were required to respond using the corresponding button. During the DV task, a target (number 9) was continuously displayed on the right of the computer screen, and a series of numerical digits were randomly displayed in the centre of the screen at $500 \mathrm{~ms}$ intervals. Participants were required to press a button whenever the centre digit and the target digit matched. The SRT and CRT had 30 trials, and the DV task had 360 trials, where 36 of those were target responses.

\section{Procedure}

All study procedures were completed in the participant's usual residence (a home or a residential care home environment) on Day 0 and from Day 2 to Day 4. Procedures were completed within a clinical research environment on Day 1 and Day 5 due to the requirements of the clinical trial, unless the participant could not travel, in which case they were assessed in their usual residence. Both follow-up assessments ( 4 and 12 weeks) were completed in the participant's usual residence.

After providing informed consent, participants completed demographic and clinical measures at baseline, which were completed with the participant's informant. Computerised attentional measures were repeated daily, at the same time of day wherever possible, between Day 1 and Day 5. The MMSE, CAMCOG, CAF and ODFAS were repeated at all follow-up stages (Day 5, 4 weeks and 12 weeks). For control participants, only baseline (Day 0) computerised attentional task data were used.

\section{Data Analysis}

Complete data were provided by a total of 36 LBD participants at baseline (23 DLB, 13 PDD), complete follow-up data were obtained from 36 participants at Day 5, from 30 participants at 4 weeks follow-up, and from 29 participants at 12 weeks follow-up. Comparator task data were obtained from 26 control participants.

Attentional task outcome measures (SRT, CRT and DV) included the percentage of correct answers, the mean reaction time (RT) to correct answers, expressed in milliseconds (ms), and the coefficient of variation (COV) of mean RTs to correct answers. The $\mathrm{COV}$ is a marker of intra-individual variability and was calculated as $\left(\mathrm{SD}_{\mathrm{RT}} / M_{\mathrm{RT}}\right) \times 100$. Two additional composite measures were calculated: firstly, the Power of Attention (PoA), which is a measure of focused attention and psychomotor/information processing speed [15], was calculated by summing SRT, CRT and DV reaction times to correct answers (ms), and secondly, the Cognitive Reaction Time (CogRT), which provides a measure of central information processing speed [15] was calculated by subtracting SRT mean RTs to correct answers from CRT mean RTs to correct answers (ms).

\section{Attentional task comparison with healthy controls}

Untransformed attentional task data (SRT, CRT, DV, PoA and $\operatorname{CogRT}$ ) were compared between LBD patients and controls using Mann-Whitney $U$ tests, with $p$ values adjusted for multiple comparisons (adjusted $p$ value $=0.005$ ).

\section{Test-retest reliability}

The test-retest reliability of attentional variables (SRT, CRT and DV \% of correct answers; SRT, CRT and DV mean reaction times to correct answers; SRT, CRT and DV coefficients of variation (COV); PoA and CogRT) was examined between baseline and Day 1 to Day 5 (short-term reliability) and between baseline, Day 5, 4 weeks and 12 weeks, to examine follow-up reliability. Only participants with complete data were included in analyses. To examine LBD test-retest reliability, all attentional variables, with the exception of the percentage of correct responses, and CogRT, were transformed using the reciprocal (1/RT) due to the non-normality of the data.

For each attentional variable, the test-retest reliability was assessed using an intra-class correlation coefficient (ICC). ICCs were calculated using a two-way mixed model with absolute agreement, based on the mean of multiple measurements [model ICC (A, 1); 25]. ICC values ranged from 0 to 1 , where low ICC values represent low levels of test-retest reliability, and high ICC values represent high levels of test-retest reliability. This was done separately for baseline and Day 1-5 data, and for baseline, 4 week and 12 week follow-up data. ICC values and $95 \%$ confidence intervals (95\% CIs) are reported for each attentional variable. Specifically, in the present study, ICC values of $<0.50$ were considered to represent poor reliability; 
values of $0.50-0.75$ represented moderate reliability; values of $0.75-0.90$ indicated good reliability, and values of $\geq 0.90$ represented excellent reliability [26]. The test-retest reliability of participant cognitive measures (MMSE, CAMCOG total and CAMCOG subscores, CAF and ODFAS) was also examined in the same manner between Day 0-5, and between Day 0, 4 weeks and 12 weeks.

\section{Additional reliability analyses}

Three additional reliability analyses were conducted. Firstly, the test-retest reliability of a reduced number of trials was examined. This was done to estimate the minimum number of trials which might be needed to obtain acceptable levels of test-retest reliability whilst minimising participant burden. To examine this, ICCs (two-way mixed models with absolute agreement $(\mathrm{A}, 1)$ ) were calculated for the reciprocal mean RT to correct answers for the CRT task, representing the task with the highest levels of test-retest reliability. This was done using blocks of 10 (ICC10) and 20 (ICC20) trials.

Secondly, to provide target sample sizes for subsequent interventional studies where objective attentional measures are desired as the primary outcome, RT measurements at baseline and 12 weeks were used to determine the standard deviation for the change in reciprocal $\mathrm{RT} \mathrm{SD}_{\text {diff }}$ between those time points. This $\mathrm{SD}_{\text {diff }}$ was used to calculate effect sizes for clinically relevant changes in RT. For a mean reaction time RT and a change of $\Delta T$, the change in reciprocal RT is given by $1 /(\mathrm{RT}-\Delta T)-1 / \mathrm{RT}$. We used the pwr package (version 1.3.0 https://cran.r-project.org/web/packages/pwr) in $R$ (R Core Team, 2019) to calculate sample size with a target power of $80 \%$ and significance level of 0.05 . This was done for CRT and PoA.

Finally, to explore whether reliability might be influenced by the severity of cognitive fluctuations, participants were divided into "low" $(n=12)$ and "high" fluctuator $(n=24)$ groups. This was done on the basis of CAF scores, where a CAF score $\leq 5$ was considered low and a CAF score of 6 and above was considered high, with the presence of clinically-significant fluctuations [27]. ICC values were calculated between Day 0-5.

\section{Results}

Baseline participant demographic and cognitive measures are summarised in Table 1.
Table 1 LBD patient baseline demographic and cognitive data $(n=36)$

\begin{tabular}{llr}
\hline & Mean & SD \\
\hline Age (years) & 75.16 & 7.96 \\
Gender (male/female) & $27(75 \%) / 9(25 \%)$ & \\
DLB/PDD & $23(63.9 \%) / 13(36.1 \%)$ & \\
UPDRS-III $^{\mathrm{a}}$ & 34.66 & 23.37 \\
MMSE & 18.03 & 6.24 \\
CAMCOG (total) & 59.19 & 21.91 \\
CAMCOG (memory subscale) & 13.36 & 6.42 \\
CAMCOG (executive function & 9.03 & 4.14 \\
$\quad$ subscale) & & \\
CAF & 2.81 & 2.97 \\
ODFAS & 3.16 & 3.24 \\
\hline
\end{tabular}

${ }^{\mathrm{a}} n=35$

\section{Comparison of data to healthy control participants}

Relative to the control group, LBD patients demonstrated significant impairments in all objective attentional variables (all $p$ values $<0.005$; Table 2 ).

\section{Short-term reliability}

The test-retest reliability of short-term attentional measures, assessed between baseline and between Day 0 and Day 5, was excellent (ICCs $>0.90$ ) for the reciprocal SRT $(\mathrm{ICC}=0.924)$, CRT $(\mathrm{ICC}=0.970)$ and DV (ICC $=0.951)$ mean RT to correct answers, as well as the reciprocal DV coefficient of variation $(\mathrm{ICC}=0.973$ ) and reciprocal PoA, which showed the highest level of reliability $(\mathrm{ICC}=0.978)$. Reciprocal $\mathrm{CRT}$ COV\% values showed good levels of reliability $(\mathrm{ICC}=0.816)$ and reciprocal SRT COV\% showed moderate levels of reliability $(I C C=0.651)$. Finally, the test-retest reliability of the CogRT was poor $(\mathrm{ICC}=0.434)$. These are summarised in Table 3. When the stability of these measures were examined in the placebo group alone, the resulting ICC values were similar (data not shown). With the exception of the CogRT measure, low and high cognitive fluctuation groups displayed comparable levels of short-term test-retest reliability (Supplementary Table 1).

\section{Follow-up reliability}

Reciprocal composite PoA measure demonstrated excellent (ICC $>0.90$ ) levels of test-retest reliability between baseline and follow-up time points (ICC $=0.927$; Table 4). Reciprocal SRT, CRT and DV reaction time to correct answers, and the CRT coefficient of variation values, 
Table 2 Control and Lewy body dementia patient Day 0 attentional task comparisons

\begin{tabular}{|c|c|c|c|c|}
\hline & \multicolumn{2}{|c|}{ Control $(n=26)$} & \multicolumn{2}{|c|}{$\operatorname{LBD}(n=36)$} \\
\hline & Mean & SD & Mean & SD \\
\hline SRT: correct answers (\%) & 100.00 & 0.00 & 85.88 & $23.14^{\mathrm{a}, * * *}$ \\
\hline SRT: mean RT (ms), correct answers & 339.57 & 59.97 & 888.66 & $776.45^{\mathrm{a}, * * *}$ \\
\hline SRT: coefficient of variation (\%) & 20.72 & 11.29 & 63.96 & $36.65^{\mathrm{a}, * * *}$ \\
\hline CRT: correct answers $(\%)$ & 97.05 & 6.49 & 68.80 & $33.76^{* * *}$ \\
\hline CRT: mean RT (ms), correct answers & 526.42 & 81.62 & 1204.13 & $1163.88^{\mathrm{a}, * * *}$ \\
\hline CRT: coefficient of variation (\%) & 18.02 & 6.93 & 58.12 & $37.55^{\mathrm{a}, * * *}$ \\
\hline DV: correct answers $(\%)$ & 96.58 & 9.76 & 58.95 & $25.83 * * *$ \\
\hline DV: mean RT (ms), correct answers & 516.39 & 52.74 & 717.97 & $155.00 * * *$ \\
\hline DV: coefficient of variation (\%) & 13.17 & 3.66 & 33.92 & $17.20 * * *$ \\
\hline PoA & 1382.38 & 143.80 & 2818.27 & $1693.47^{\mathrm{b}, * * *}$ \\
\hline CogRT & 186.85 & 59.06 & 355.38 & $1192.64^{\mathrm{b}, * * *}$ \\
\hline
\end{tabular}

$S D$ standard deviation, $S R T$ simple reaction time, $R T$ reaction time, $C R T$ choice reaction time, $D V$ digit vigilance

${ }^{\mathrm{a}} n=34$; due to incomplete data

${ }^{\mathrm{b}} n=32$; due to incomplete data

${ }^{* * *} p<.001$ (control vs. LBD)
Table 3 Test-retest reliability of Day 0 to Day 5 attentional measures

\begin{tabular}{|c|c|c|}
\hline Attentional measure & ICC & $95 \% \mathrm{CI}$ \\
\hline SRT: correct answers $(\%)^{\mathrm{a}}$ & 0.692 & $(0.491-0.833)$ \\
\hline SRT: $1 /$ mean RT to correct answers ${ }^{\mathrm{a}}$ & 0.924 & $(0.873-0.959)$ \\
\hline SRT: $1 /$ coefficient of variation $(\%)^{\mathrm{a}}$ & 0.651 & $(0.419-0.811)$ \\
\hline CRT: correct answers $(\%)^{\mathrm{b}}$ & 0.770 & $(0.487-0.893)$ \\
\hline CRT: $1 /$ mean RT to correct answers ${ }^{\mathrm{a}}$ & 0.970 & $(0.950-0.984)$ \\
\hline CRT: $1 /$ coefficient of variation $(\%)^{\mathrm{a}}$ & 0.819 & $(0.701-0.902)$ \\
\hline DV: correct answers $(\%)^{\mathrm{a}}$ & 0.898 & $(0.830-0.945)$ \\
\hline DV: $1 /$ mean RT, correct answers ${ }^{\mathrm{c}}$ & 0.951 & $(0.918-0.974)$ \\
\hline DV: $1 /$ coefficient of variation $(\%)^{\mathrm{d}}$ & 0.973 & $(0.652-0.890)$ \\
\hline $1 / \mathrm{PoA}^{\mathrm{e}}$ & 0.978 & $(0.963-0.989)$ \\
\hline $\operatorname{CogRT}{ }^{d}$ & 0.434 & $(0.042-0.702)$ \\
\hline
\end{tabular}

${ }^{\mathrm{a}} n=31$

$\mathrm{b}_{n=33}$

${ }^{c} n=30$

${ }^{\mathrm{d}} n=29$

${ }^{\mathrm{e}} n=27$

showed good levels of test-retest reliability (ICCs $\geq 0.85$ ) whereas reciprocal SRT and DV coefficient of variations, and the composite CogRT measure, all displayed poor levels of test-retest reliability (all ICCs $<0.32$ ).

\section{Test-retest reliability of cognitive measures}

Short-term levels of test-retest reliability were poor for the CAF (ICC $=0.223)$; moderate for the ODFAS ( $\mathrm{ICC}=0.543$ ), good for CAMCOG memory and executive
Table 4 Test-retest reliability of Day 0 and follow-up (Day 5, 4 and 12 weeks) attentional measures

\begin{tabular}{|c|c|c|}
\hline Attentional measure & ICC & $95 \% \mathrm{CI}$ \\
\hline SRT: correct answers $(\%)^{\mathrm{a}}$ & 0.895 & $(0.805-0.950)$ \\
\hline SRT: $1 /$ mean RT to correct answers ${ }^{b}$ & 0.849 & $(0.700-0.931)$ \\
\hline SRT: $1 /$ coefficient of variation $(\%)^{c}$ & 0.277 & $(-0.481-0.674)$ \\
\hline CRT: correct answers $(\%)^{\mathrm{d}}$ & 0.896 & $(0.810-0.950)$ \\
\hline CRT: $1 /$ mean RT to correct answers ${ }^{\mathrm{c}}$ & 0.893 & $(0.780-0.953)$ \\
\hline CRT: $1 /$ coefficient of variation $(\%)^{\mathrm{b}}$ & 0.874 & $(0.743-0.945)$ \\
\hline DV: correct answers $(\%)^{\mathrm{b}}$ & 0.843 & $(0.706-0.926)$ \\
\hline DV: $1 /$ mean RT, correct answers ${ }^{\mathrm{c}}$ & 0.766 & $(0.539-0.892)$ \\
\hline DV: $1 /$ coefficient of variation $(\%)^{b}$ & 0.159 & $(-0.607-0.606)$ \\
\hline $1 / \mathrm{PoA}^{\mathrm{e}}$ & 0.927 & $(0.846-0.969)$ \\
\hline $\operatorname{CogRT} \mathrm{T}^{\mathrm{e}}$ & 0.314 & $(-0.459-0.709)$ \\
\hline
\end{tabular}

${ }^{\mathrm{a}} n=24$

${ }^{\mathrm{b}} n=23$

${ }^{\mathrm{c}} n=21$

$\mathrm{d}_{n=25}$

${ }^{\mathrm{e}} n=20$

subscales (ICCs $=0.884$ and 0.846$)$ and excellent for the MMSE and CAMCOG total (ICCs $=0.932 \& 0.938$; Supplementary Table 2). Between baseline, and 4-week and 12-week follow-up time points, the MMSE, CAMCOG total, CAMCOG memory and CAMCOG executive function subscales all displayed excellent test-retest reliability levels (all ICCs $\geq 0.90$ ), and the CAF and ODFAS both displayed good reliability levels (ICCs $=0.818$ and 0.719 respectively; Supplementary Table 3). 


\section{Minimum number of trials}

ICC values, calculated on the basis of a shortened version of the CRT between Day 0 and Day 5, showed excellent levels of reliability for both 10 and 20 individual trials for the reciprocal mean RT to correct answers: ICC10 $=0.945$ (95\% CI 0.907-0.972); ICC20 $=0.965$ (95\% CI 0.941-0.982).

\section{Target sample sizes}

Based on a hypothetical within-participants study where attentional measures were the primary outcome measure, the SD on the difference in score between Day 0 and 12 weeks was $3.8 \times 10^{-4} \mathrm{~s}^{-1}$ for CRT and $9.3 \times 10^{-5} \mathrm{~s}^{-1}$ for PoA. To observe a change in CRT from $1204 \mathrm{~ms}$ (Table 1) over 12 weeks, the estimated required sample size at $80 \%$ power for a change of $100 \mathrm{~ms}$ is $n=200 ; 200 \mathrm{~ms}$ would require $n=43$, and for a change of $300 \mathrm{~ms}, n=17$. For PoA, with a mean baseline of $2818 \mathrm{~ms}$, the required sample size for a change of 200, 400, or $600 \mathrm{~ms}$ would be $n=95, n=22$ or $n=10$ respectively.

\section{Discussion}

Contrary to expectations, there were high levels of test-retest reliability for some, but not all, attentional measures. This was the case at short-term and followup time points. Specifically, overall reaction times and intra-individual reaction time variability displayed high test-retest reliability in the short-term (Baseline and Day 1-5) and between baseline and follow-up time points (4 and 12 weeks). The composite Power of Attention measure showed high test-retest reliability, but cognitive reaction time, as a marker of central information processing speed, showed a poor test-retest reliability. These findings are not in line with the hypotheses that individuals would demonstrate poor test-retest reliability in: (1) overall reaction times; (2) intra-individual reaction time variability and (3) composite measures of focussed attention and central information processing speed, at both short-term and longer-term follow-up. Potential reasons for this include the possibility that whilst objectively, patients with LBD demonstrate high levels of intra-individual variability, participants may have had the same relative degree of objective attentional instability, resulting in a minimal impact upon attentional measures [10-12]. Finally, all patients were taking medications including cholinesterase inhibitors, which can affect attention [15]. However, medication withdrawal prior to study entry was not feasible given the trial design and the potential for the clinical deterioration of participants. Overall, these results still indicate that in the short-term, attentional measures which are commonly used in LBD clinical trials, generally display very high levels of test-retest reliability, even at 12 weeks follow-up. These findings have important implications for the design and delivery of LBD clinical trials using these measures as outcomes, as our study suggests that many of these are reliable.

These findings indicate that the reciprocal Power of Attention composite measure displayed excellent levels of reliability, and appears to be a very robust marker of cognition in LBD, even at follow-up time points of up to 12 weeks. Although a period of 12 weeks is in line with previous pharmacological clinical trials in LBD, and the findings of the present study are relevant to trial design [28, 29], future studies should specifically examine whether these high reliability levels persist beyond 12 weeks. This is important as within clinical trials, it is common to assess patients over a longer period of time; for example, LBD trials report followup time points of approximately 6 months $[15,30]$ or 1 year [31]. Subjective declines in cognition, with a large degree of variation, have been observed over longer time periods in LBD [32] and this might also be the case with objective measures. Another important finding in the present study was that objective attentional measures displayed comparable levels of reliability to the MMSE and CAMCOG. Given the ease and standardisation of objective test administration, these may be suitable trial outcome measures.

In terms of subjective measures, the poor short-term reliability of the CAF may reflect its intended use as a measurement tool for cognitive fluctuations in the preceding 4 weeks [23]. However, the ODFAS demonstrated moderate shortterm reliability, and good longer-term reliability, which is surprising as this should only measure cognitive fluctuations in the preceding day. This suggests that the development of alternative measures may be needed to assess cognitive fluctuations subjectively over shorter time spans in heterogeneous LBD patient groups.

The additional analyses conducted in the present study also have important implications for LBD trial design. Firstly, we demonstrated that for the CRT task, between baseline and Day 5, the use of a reduced number of trials (10 or 20, compared to the standard 30) can still result in excellent test-retest reliability. This suggests that shorter duration tasks are feasible, which would reduce participant burden whilst maintaining reliability. Future work should examine if this applies to all commonly-used reaction time measures, and if this is affected by participant heterogeneity (e.g. medication use, dementia severity). The present study also provides indicative minimum sample sizes to account for the test-retest reliability of attentional outcome measures, where comparable improvements in reaction time are expected. For instance, in pharmacological studies, CRT improvements of 100-200 ms have been observed in a trial of memantine, and PoA improvements of approximately $300 \mathrm{~ms}$ in a trial 
of rivastigmine, relative to placebo, both at 24 weeks [15, 33]. Therefore, these results can directly inform the design of future LBD clinical trials.

Overall, these findings should be replicated in a larger sample size, where this is the primary aim of the study, to confirm the test-retest reliability of these attentional measures. Future studies should ensure that they have sufficient statistical power to be able to examine reliability at followup stages, as although we have demonstrated that demanding clinical trials, with daily assessments, are feasible in an LBD population [19], in the present study, a relatively large number of participants (approximately 20\% of participants at 12 weeks) were lost to follow-up, although this consistent with other LBD trials [e.g. 30]. Finally, future studies should also examine whether the attentional measures which were used in the present study could be further optimised. Whilst it was beyond the scope of the present study, future work should assess the utility of attentional testing in the measurement of cognitive fluctuations. Whilst difficult to define, cognitive fluctuations are believed to consist of a cognitive component, which is apparent in the DLB-specific attentional and working memory dysfunction, and an arousal component, which can influence attention [7, 34-37]. Therefore, studies should examine if the cognitive and arousal components independently, or additively, contribute to the symptom of cognitive fluctuations, as this may result in better objective measurement tools.

Specific strengths of the current study include the comprehensive and well-characterised nature of the patients, as the LBD diagnosis was made by two highly-experienced clinicians; additionally, participants demonstrated medication stability before entering the study. This is particularly important as reaction time measures are very sensitive to the effects of a range of pharmacological agents. A further strength of this study is in the fact that the participants included in the trial had a wide range of dementia severity. This included participants who demonstrated high impairment, thus maximising the generalisability of the results due to the participant heterogeneity.

Despite the important implications of the present study, there are several potential limitations. The main limitation is in the post hoc secondary analysis of clinical trial data; however, persuading participants to take part in an intensive observational study of this nature may be practically difficult, particularly when the participants have a range of dementia severity. Additionally, we could not compare test-retest reliability between DLB and PDD participants due to the lack of statistical power. However, both patient groups display similar levels of attentional impairment [5, 6]. Whilst there may be domain-specific differences between DLB and PDD in the rate of cognitive decline over time, it is not known if this applies to objective attention [38] and future studies may wish to assess whether this can influence attentional reliability. Additionally, although minor variations in time between baseline and Day 1 data collection may have influenced the results, given the excellent levels of reliability observed, this is likely to have had only a minimal effect and was controlled for by repeating the baseline tests where the delay was judged to be excessive.

A further limitation is that these data were obtained from participants from a trial investigating the effects of tDCS upon visual hallucinations. Although attentional measures were not the primary outcome measure in the intervention group, tDCS may affect brain networks beyond the targeted localised region of interest [39]. It is extremely unlikely that this would have affected the results of the present study, since attentional brain regions were not targeted, and several variables can affect tDCS efficacy in dementia populations [40]. Nonetheless, future prospective studies should specifically examine the reliability of these measures outside of intervention studies. Additionally, due to the absence of patients without cognitive fluctuations, it was not possible to specifically compare the reliability between patients with, and without, cognitive fluctuations. Although our results suggest that cognitive fluctuation severity may not influence the reliability of reaction time measures, with the exception of the composite Cognitive Reaction Time, at least in the short-term and when patients are divided on the basis of whether fluctuations are, or are not, clinically-significant, further work is needed to confirm this. Finally, due to data non-normality, we could not assess the reliability of untransformed data, with the exception of the CRT. Further studies, with larger sample sizes, should examine untransformed data reliability, since most LBD trials report untransformed data [24]. However, in the context of LBD trials, the transformation of data does not present any technical challenges.

In conclusion, several computerised attentional measures show excellent levels of test-retest reliability. The reciprocal composite Power of Attention measure displays the highest test-retest reliability, both short-term, and up to 12 weeks follow-up.

Supplementary Information The online version contains supplementary material available at https://doi.org/10.1007/s00415-022-10977-4.

Acknowledgements We would like to thank all study participants, including people with dementia, and their families and/or carers. We would also like to thank Dr. Barbara Wilson and the staff of North East Dementia and Neurodegenerative Diseases Network (DeNDRoN) for their assistance with participant recruitment, and Dr. Charlotte Allan and Dr. Sophia Bennett for verifying patient diagnoses, and Dr. Alison Killen and Steven Errington for their assistance with collecting data.

Funding This work was financially supported by the NIHR Newcastle Biomedical Research Centre awarded to the Newcastle upon Tyne Hospitals NHS Foundation Trust and Newcastle University, and by Northumberland, Tyne and Wear NHS Foundation Trust Research Capability Funding. 
Data availability The data that support the findings of this study are available from the corresponding author upon reasonable request.

\section{Declarations}

Conflicts of interest The authors declare that they have no conflict of interest.

Ethical standards The authors assert that all procedures contributing to this work comply with the ethical standards of the relevant national and institutional committees on human experimentation and with the Helsinki Declaration of 1964, as revised in 2008

Open Access This article is licensed under a Creative Commons Attribution 4.0 International License, which permits use, sharing, adaptation, distribution and reproduction in any medium or format, as long as you give appropriate credit to the original author(s) and the source, provide a link to the Creative Commons licence, and indicate if changes were made. The images or other third party material in this article are included in the article's Creative Commons licence, unless indicated otherwise in a credit line to the material. If material is not included in the article's Creative Commons licence and your intended use is not permitted by statutory regulation or exceeds the permitted use, you will need to obtain permission directly from the copyright holder. To view a copy of this licence, visit http://creativecommons.org/licenses/by/4.0/.

\section{References}

1. Vann Jones SA, O'Brien JT (2014) The prevalence and incidence of dementia with Lewy bodies: a systematic review of population and clinical studies. Psychol Med 44(4):673-683. https://doi.org/ 10.1017/S0033291713000494

2. McKeith IG, Boeve BF, Dickson DW, Halliday G, Taylor JP, Weintraub D et al (2017) Diagnosis and management of dementia with Lewy bodies: fourth consensus report of the DLB consortium. Neurology. https://doi.org/10.1212/WNL.0000000000 004058

3. Aarsland D, Andersen K, Larsen JP, Lolk A (2003) Prevalence and characteristics of dementia in parkinson disease: an 8-year prospective study. Arch Neurol 60(3):387-392. https://doi.org/ 10.1001/archneur.60.3.387

4. Hely MA, Reid WGJ, Adena MA, Halliday GM, Morris JGL (2008) The Sydney multicenter study of Parkinson's disease: the inevitability of dementia at 20 years. Mov Disord 23(6):837-844. https://doi.org/10.1002/mds.21956

5. Emre M, Aarsland D, Brown R, Burn DJ, Duyckaerts C, Mizuno $Y$ et al (2007) Clinical diagnostic criteria for dementia associated with Parkinson's disease. Mov Disord 22(12):1689-1707. https:// doi.org/10.1002/mds.21507

6. Lippa CF, Duda JE, Grossman M, Hurtig HI, Aarsland D, Boeve BF et al (2007) DLB and PDD boundary issues: diagnosis, treatment, molecular pathology, and biomarkers. Neurology 68(11):812-819. https://doi.org/10.1212/01.wnl.0000256715. 13907.d3

7. Bradshaw J, Saling M, Hopwood M, Anderson V, Brodtmann A (2004) Fluctuating cognition in dementia with Lewy bodies and Alzheimer's disease is qualitatively distinct. J Neurol Neurosurg Psychiatry 75(3):382-387

8. Metzler-Baddeley C (2007) A review of cognitive impairments in dementia with Lewy bodies relative to Alzheimer's disease and
Parkinson's disease with dementia. Cortex 43(5):583-600. https:// doi.org/10.1016/S0010-9452(08)70489-1

9. Ferman TJ, Smith GE, Boeve BF, Graff-Radford NR, Lucas JA, Knopman DS et al (2006) Neuropsychological differentiation of dementia with Lewy bodies from normal aging and Alzheimer's disease. Clin Neuropsychol 20(4):623-636. https://doi.org/10. 1080/13854040500376831

10. Ballard C, O'Brien J, Gray A, Cormack F, Ayre G, Rowan E et al (2001) Attention and fluctuating attention in patients with dementia with Lewy bodies and Alzheimer disease. Arch Neurol 58(6):977-982. https://doi.org/10.1001/archneur.58.6.977

11. Firbank M, Kobeleva X, Cherry G, Killen A, Gallagher P, Burn DJ et al (2016) Neural correlates of attention-executive dysfunction in Lewy body dementia and Alzheimer's disease. Hum Brain Mapp 37(3):1254-1270. https://doi.org/10.1002/hbm.23100

12. Schumacher J, Cromarty R, Gallagher P, Firbank MJ, Thomas AJ, Kaiser M et al (2019) Structural correlates of attention dysfunction in Lewy body dementia and Alzheimer's disease: an exGaussian analysis. J Neurol 266(7):1716-1726. https://doi.org/10. 1007/s00415-019-09323-y

13. Bronnick K, Ehrt U, Emre M, De Deyn PP, Wesnes K, Tekin S et al (2006) Attentional deficits affect activities of daily living in dementia-associated with Parkinson's disease. J Neurol Neurosurg Psychiatry 77(10):1136-1142. https://doi.org/10.1136/jnnp.2006. 093146

14. Shine JM, Halliday GM, Naismith SL, Lewis SJ (2011) Visual misperceptions and hallucinations in Parkinson's disease: dysfunction of attentional control networks? Mov Disord 26(12):2154 2159. https://doi.org/10.1002/mds.23896

15. Wesnes KA, Aarsland D, Ballard C, Londos E (2015) Memantine improves attention and episodic memory in Parkinson's disease dementia and dementia with Lewy bodies. Int J Geriatr Psychiatry 30(1):46-54. https://doi.org/10.1002/gps.4109

16. Rowan E, McKeith IG, Saxby BK, O'Brien JT, Burn D, Mosimann U et al (2007) Effects of donepezil on central processing speed and attentional measures in Parkinson's disease with dementia and dementia with Lewy bodies. Dement Geriatr Cogn Disord 23(3):161-167. https://doi.org/10.1159/000098335

17. Wesnes KA, McKeith I, Edgar C, Emre M, Lane R (2005) Benefits of rivastigmine on attention in dementia associated with Parkinson disease. Neurology 65(10):1654-1656. https://doi.org/10.1212/01. wnl.0000184517.69816.e9

18. Mueller C, Ballard C, Corbett A, Aarsland D (2017) The prognosis of dementia with Lewy bodies. The Lancet Neurology 16(5):390-398. https://doi.org/10.1016/S1474-4422(17)30074-1

19. Elder GJ, Colloby SJ, Firbank MJ, McKeith IG, Taylor JP (2019) Consecutive sessions of transcranial direct current stimulation do not remediate visual hallucinations in Lewy body dementia: a randomised controlled trial. Alzheimers Res Ther 11(1):9. https:// doi.org/10.1186/s13195-018-0465-9

20. Folstein MF, Folstein SE, McHugh PR (1975) "Mini-mental state": A practical method for grading the cognitive state of patients for the clinician. J Psychiatr Res 12(3):189-198. https:// doi.org/10.1016/0022-3956(75)90026-6

21. Goetz CG, Tilley BC, Shaftman SR, Stebbins GT, Fahn S, Martinez-Martin P et al (2008) movement disorder society-sponsored revision of the unified Parkinson's disease rating scale (MDSUPDRS): scale presentation and clinimetric testing results. Mov Disord 23(15):2129-2170. https://doi.org/10.1002/mds.22340

22. Roth M, Tym E, Mountjoy CQ, Huppert FA, Hendrie H, Verma $S$ et al (1986) CAMDEX. A standardised instrument for the diagnosis of mental disorder in the elderly with special reference to the early detection of dementia. Br J Psychiatry 149:698-709

23. Walker MP, Ballard CG, Ayre GA, Wesnes K, Cummings JL, McKeith IG et al (2000) The clinician assessment of fluctuation and the one day fluctuation assessment scale: two methods 
to assess fluctuating confusion in dementia. Br J Psychiatry 177(3):252-256. https://doi.org/10.1192/bjp.177.3.252

24. Wesnes KA, McKeith IG, Ferrara R, Emre M, Del Ser T, Spano $\mathrm{PF}$ et al (2002) Effects of rivastigmine on cognitive function in dementia with lewy bodies: a randomised placebo-controlled international study using the cognitive drug research computerised assessment system. Dement Geriatr Cogn Disord 13(3):183-192. https://doi.org/10.1159/000048651

25. Liljequist D, Elfving B, Skavberg RK (2019) Intraclass correlation-a discussion and demonstration of basic features. PLoS ONE 14(7):e0219854. https://doi.org/10.1371/journal.pone.02198 54

26. Koo TK, Li MY (2016) A guideline of selecting and reporting intraclass correlation coefficients for reliability research. J Chiropr Med 15(2):155-163. https://doi.org/10.1016/j.jcm.2016.02.012

27. Walker MP, Ayre GA, Cummings JL, Wesnes K, McKeith IG, O'Brien JT et al (2000) Quantifying fluctuation in dementia with Lewy bodies, Alzheimer's disease, and vascular dementia. Neurology 54(8):1616-1625

28. Mori E, Ikeda M, Kosaka K, Donepezil DLBSI (2012) Donepezil for dementia with Lewy bodies: a randomized, placebo-controlled trial. Ann Neurol 72(1):41-52. https://doi.org/10.1002/ana.23557

29. Murata M, Odawara T, Hasegawa K, Kajiwara R, Takeuchi H, Tagawa $\mathrm{M}$ et al (2020) Effect of zonisamide on parkinsonism in patients with dementia with Lewy bodies: a phase 3 randomized clinical trial. Parkinsonism Relat Disord 76:91-97. https://doi.org/ 10.1016/j.parkreldis.2019.12.005

30. Aarsland D, Ballard C, Walker Z, Bostrom F, Alves G, Kossakowski K et al (2009) Memantine in patients with Parkinson's disease dementia or dementia with Lewy bodies: a double-blind, placebo-controlled, multicentre trial. Lancet Neurol 8(7):613-618. https://doi.org/10.1016/S1474-4422(09)70146-2

31. Ikeda M, Mori E, Kosaka K, Iseki E, Hashimoto M, Matsukawa N et al (2013) Long-term safety and efficacy of donepezil in patients with dementia with Lewy bodies: results from a 52-week, openlabel, multicenter extension study. Dement Geriatr Cogn Disord 36(3-4):229-241. https://doi.org/10.1159/000351672

32. Kramberger MG, Auestad B, Garcia-Ptacek S, Abdelnour C, Olmo JG, Walker $\mathrm{Z}$ et al (2017) Long-term cognitive decline in dementia with lewy bodies in a large multicenter, international cohort. J Alzheimers Dis 57(3):787-795. https://doi.org/10.3233/ JAD-161109

33. Emre M, Aarsland D, Albanese A, Byrne EJ, Deuschl G, De Deyn $\mathrm{PP}$ et al (2004) Rivastigmine for dementia associated with Parkinson's disease. N Engl J Med 351(24):2509-2518. https://doi.org/ 10.1056/NEJMoa041470

34. McKeith IG, Dickson DW, Lowe J, Emre M, O’Brien JT, Feldman $\mathrm{H}$ et al (2005) Diagnosis and management of dementia with Lewy bodies: third report of the DLB consortium. Neurology 65(12):1863-1872. https://doi.org/10.1212/01.wnl.0000187889. 17253.b1

35. McKeith IG (2006) Consensus guidelines for the clinical and pathologic diagnosis of dementia with Lewy bodies (DLB): report of the Consortium on DLB International Workshop. J Alzheimers Dis 9(3):417-423

36. Ferman TJ, Smith GE, Boeve BF, Ivnik RJ, Petersen RC, Knopman D et al (2004) DLB fluctuations: specific features that reliably differentiate DLB from AD and normal aging. Neurology 62(2):181-187

37. Morandi A, Davis D, Bellelli G, Arora RC, Caplan GA, Kamholz $B$ et al (2017) The diagnosis of delirium superimposed on dementia: an emerging challenge. J Am Med Dir Assoc 18(1):12-18. https://doi.org/10.1016/j.jamda.2016.07.014

38. Smirnov DS, Galasko D, Edland SD, Filoteo JV, Hansen LA, Salmon DP (2020) Cognitive decline profiles differ in Parkinson disease dementia and dementia with Lewy bodies. Neurology 94(20):e2076-e2087. https://doi.org/10.1212/WNL.0000000000 009434

39. To WT, De Ridder D, Hart J Jr, Vanneste S (2018) Changing brain networks through non-invasive neuromodulation. Front Hum Neurosci 12:128. https://doi.org/10.3389/fnhum.2018.00128

40. Elder GJ, Taylor JP (2014) Transcranial magnetic stimulation and transcranial direct current stimulation: treatments for $\operatorname{cog}$ nitive and neuropsychiatric symptoms in the neurodegenerative dementias? Alzheimers Res Ther 6(9):74. https://doi.org/10.1186/ s13195-014-0074-1 